\title{
Cancer patients in com plementary and alternative medicine: More harm
}

\author{
Abish Adhikari ${ }^{1}$, MD; Rupendra Bahadur Adhikari², PhD \\ ${ }^{1}$ Department of Radiation Onchology, Kathmandu Cancer Hospital, Bhaktapur, Nepal \\ ${ }^{2}$ Department of Neuro Sciences, Grande International Hospital, Kathmandu, Nepal
}

The aim of treating cancer patients is:

To cure sometimes,

To relieve often,

To comfort always!

The diagnosis of cancer comes as a surprise to most patients and their families with an initial period of denial. Cancer being a chronic disease usually with a fatal outcome, is often considered a death sentence.

With modern treatment modalities like precise radiotherapy, newer molecules of chemotherapy and specialized surgical techniques, many cancers are now curable if found at an early stage. Sadly, many cancer patients in Nepal reach the oncologist in advanced stages, hence the treatment may not be as effective ${ }^{1}$.

The treatment strategy for advanced cancer is complicated - with a combination of surgery, chemotherapy, radiotherapy - which comes at a cost - both financial and clinical. The clinical cost being the burden of toxicities that comes with treatment. Due to wrong depiction in social media, cinemas and public talks, cancer treatment is often considered toxic - a mix of fear of side effects, and the disbelief that has been curated in the society since ages.

On the other hand, Complementary and Alternative Medicines (CAMs) Practitioners claim a 'mystical solution' to the serious problem with powders, capsules and food products. Advertised to be 'without side effects', cancer patients tend to believe them more - although those products have no scientific backing or data to prove their efficacy.

CAMs are defined as practices, health care systems, or products that cannot be considered as conventional medicine. These practices include diverse procedures like osteopathy, homeopathy, acupuncture, healing touch, and naturopathy².
In Nepal, there are areas as remote where healthcare services are not accessible which leaves people with no choice but to take the help of the shaman, witch doctors and faith healers. On the contrary, this tendency to believe in 'magical treatment' exists even in urban areas where proper healthcare services exist!

In recent times, 'alternative medicine' centers have been mushrooming because they do not require professional licensing or any academic certifications. Newspapers and Radio stations keep broadcasting their advertisements claiming a perfect solution of all ailments including cancer.

Sanford et al. recently published a data ${ }^{3}$ in JAMA that found that at least a third of cancer patients in the United States use Complementary and alternative medicines. Majority of them were using herbal medicines in an attempt to get better.

Gras et al. in France found that $83.0 \%$ of cancer patients included in the study had used Complementary and Alternative Medicines (CAMs) at least once since the beginning of their anticancer treatment. CAMs ranged from osteopathy, homeopathy, acupuncture, healing touch, magnetism, naturopathy, suction cups, Chinese medicine, reflexology to hypnosis ${ }^{2}$. Out of the 200 patients enrolled, only $5 \%$ of the patients had been taking CAMs as the primary treatment of cancer whereas $83 \%$ were taking it to reduce sideeffects of cancer treatment.

Although there is no detailed data from Nepal, we believe that the number of cancer patients taking CAMs instead of proper cancer treatment is much more than that reported from the developed world. In developed countries, CAMs are usually taken as a complementary treatment along with modern cancer treatment. Whereas in Nepal, the CAMs practitioners claim their speciality to be the primary treatment for cancer with no proper 
evidence. This puts many patients in confidence to avoid the proven modern medicine cancer treatment and resort to CAMs which unfortunately leads to progression of the disease.

Many clinicians encounter patients who have spent hefty amounts on alternative medicine which hasn't proved beneficial. We often see cancer patients who were diagnosed at a very early curable stage but reach the oncologist at a very late incurable stage because they chose to go to 'we cure everything without side-effects' CAMs practitioners.

Almost all cancer types have a significantly reduced survival when the stage goes up, and thus starting the cancer specific treatment at its early stage is crucial. Delaying the initiation of breast cancer chemotherapy for just three months instead of within a month was found to have a higher mortality rate in one of the detailed analysis of treatment delays ${ }^{4}$.

Delays between diagnosis and initial treatment were associated with worsened overall survival for stages I and II breast, lung, renal, and pancreatic cancers, and stage II colorectal cancer, with increased risk of mortality of $1.2 \%$ to $3.2 \%$ per week of delay, adjusting for comorbidities and other variables ${ }^{5}$.

Although plant extracts are the major source of chemotherapeutic agents ${ }^{6}$, it doesn't mean taking them directly would help patients in reducing the tumor burden. There are few case reports but no scientifically credible data sets to prove that using plants in their natural form helps in cancer management.

The governing bodies should take immediate action to prevent more cancer patients from being misinformed and misguided about the CAMs. Cancer patients have hope of cure but the time window to attain the best results is limited, meaning the treatment with surgerychemotherapy-radiotherapy should start as soon as possible. When patients of cancer linger around CAMs, it only leads to disease progression and the treatment plan often changes from curative to palliative which has a grave prognosis.

\section{References:}

1. Piya MK, Acharya SC. Oncology in Nepal. South Asian J Cancer. 2012;1(1):5-8.

2. Gras, M., Vallard, A., Brosse, C., Beneton, A., Sotton, S., Guyotat, D., Fournel, P., Daguenet, E., Magné, N. and Morisson, S. Use of Complementary and Alternative Medicines among Cancer Patients: A Single-Center Study. Oncology. 2019; 97(1):18-25.

3. Sanford NN, Sher DJ, Ahn C, Aizer AA, Mahal BA. Prevalence and Nondisclosure of Complementary and Alternative Medicine Use in Patients With Cancer and Cancer Survivors in the United States. JAMA Oncol. 2019;5(5):735-7.

4. Bleicher RJ, Ruth K, Sigurdson ER, et al. Time to Surgery and Breast Cancer Survival in the United States. JAMA Oncol. 2016;2(3):3309.

5. Khorana, Alok $A$ et al. Time to initial cancer treatment in the United States and association with survival over time: An observational study. PLoS One vol. 14,3 e0213209.

6. Cragg G, Newman D. Plants as a source of anti-cancer agents. J Ethnopharmacol. 2005;100(1-2):72-9. 